\title{
Multi-Energy Gamma-Ray Attenuations for Non-Destructive Detection of Hazardous Materials
}

\author{
Kaylyn Olshanoski, Chary Rangacharyulu \\ Department of Physics and Engineering Physics, University of Saskatchewan, Saskatoon, Canada \\ Email: chary.r@usask.ca
}

How to cite this paper: Olshanoski, K. and Rangacharyulu, C. (2022) Multi-Energy Gamma-Ray Attenuations for Non-Destructive Detection of Hazardous Materials. Journal of Modern Physics, 13, 66-80. https://doi.org/10.4236/jmp.2022.131005

Received: November 24, 2021

Accepted: January 23, 2022

Published: January 26, 2022

Copyright $\odot 2022$ by author(s) and Scientific Research Publishing Inc. This work is licensed under the Creative Commons Attribution International License (CC BY 4.0).

http://creativecommons.org/licenses/by/4.0/

\section{(c) (i) Open Access}

\begin{abstract}
We present a non-destructive method (NDM) to identify minute quantities of high atomic number $(Z)$ elements in containers such as passenger baggage, goods carrying transport trucks, and environmental samples. This method relies on the fact that photon attenuation varies with its energy and properties of the absorbing medium. Low-energy gamma-ray intensity loss is sensitive to the atomic number of the absorbing medium, while that of higher-energies vary with the density of the medium. To verify the usefulness of this feature for NDM, we carried out simultaneous measurements of intensities of multiple gamma rays of energies 81 to $1408 \mathrm{keV}$ emitted by sources ${ }^{133} \mathrm{Ba}$ (half-life $=10.55 \mathrm{y}$ ) and ${ }^{152} \mathrm{Eu}$ (half-life $=13.52 \mathrm{y}$ ). By this arrangement, we could detect minute quantities of lead and copper in a bulk medium from energy dependent gamma-ray attenuations. It seems that this method will offer a reliable, low-cost, low-maintenance alternative to X-ray or accelerator-based techniques for the NDM of high-Z materials such as mercury, lead, uranium, and transuranic elements etc.
\end{abstract}

\section{Keywords}

Non-Destructive Detection, Multi-Energy Photons, Radioactive Sources, Intensity Measurements, Safety and Security, XCOM Calculations

\section{Introduction}

Contaminations of high atomic number $(Z)$ materials are of concern for both security and health reasons [1] [2]. Governments and industries are paying much attention to detecting these elements in goods during transportation or present in our surroundings, either water resources or agricultural environments. Cur- 
rently, nuclear detection techniques mostly involve the use of X-ray beams of a few hundred $\mathrm{keV}$, while high-energy gamma rays, or neutron beams from particle accelerators are considered as candidate tools [1] [2]. However, the latter technologies involve high-energy accelerators requiring high maintenance, large real estate, and the associated high costs of infrastructure and operations. They are also beset by the low yields of nuclear interactions. In this work, we suggest that multi-energy gamma-ray tomography, making use of photon emissions of radioactive isotopes of a few years of half-life, offers a promising tool. Below, we present the background physics, our choice of radioactive isotopes for the current work, and experimental data with a single detector to mimic a traveler's suitcase inspection and comparison of the experimental data with the standard theoretical XCOM database [3] results, which can be extended to a tomographic setting. We also present the results of our XCOM based calculation for plutonium contraband material smuggling and mercury contamination in water.

\section{Background Physics}

As is the case with X-rays, multi-energy gamma-ray tomography relies on the variation of photon intensity attenuation coefficients in material media. The intensity $I$ of photons of energy $E_{\gamma}$ as they pass through a thickness $x$ in a material medium of density $\rho$ and atomic number $Z$ can be written as:

$$
I\left(E_{\gamma}, Z, \rho, x\right)=I_{0} \mathrm{e}^{-\mu_{\rho}\left(E_{\gamma}, Z\right) \cdot \rho x}
$$

where $I_{0}$ is the intensity at the entrance of the medium, $\mu_{\rho}\left[\mathrm{cm}^{2} / \mathrm{g}\right]$ is the mass attenuation coefficient of the medium which varies with photon energy. It should be noted that the mass attenuation coefficient $\mu_{\rho}$ is specific to the atomic number of the medium and the photon energy. It does not depend on the density nor on the physical state (solid, liquid or gas) of the medium. The mass attenuation coefficients for pure elements can be found for energies in the range $1 \mathrm{keV}-100 \mathrm{GeV}$ from the XCOM database [4], which can be easily extended to compounds and mixtures through Bragg's formula [5]. XCOM also provides the data for compounds and mixtures.

In Figure 1, we plot the mass attenuation coefficients of $1 \mathrm{keV}-10 \mathrm{MeV}$ photons for six elements: carbon $(Z=6)$, oxygen $(Z=8)$, aluminum $(Z=13)$, copper $(Z=29)$, tin $(Z=50)$ and lead $(Z=82)$ covering nearly the entire periodic table of stable materials of interest. Two conspicuous features present themselves: 1) for low energies of $E_{\gamma}<0.1 \mathrm{MeV}$, the mass attenuation coefficients vary by a factor of about $10-100$, and they exhibit discontinuities characteristic of absorption edges of the elements, 2) the coefficients are nearly constant for $1<E_{\gamma}[\mathrm{MeV}]<10$, with an overall change in the attenuation coefficients of less than three for the entire periodic table. These features indicate that the attenuation of gamma rays of $E_{\gamma}>1 \mathrm{MeV}$ in a medium of certain linear thickness is not sensitive to the elemental composition, but it is strongly affected by density 


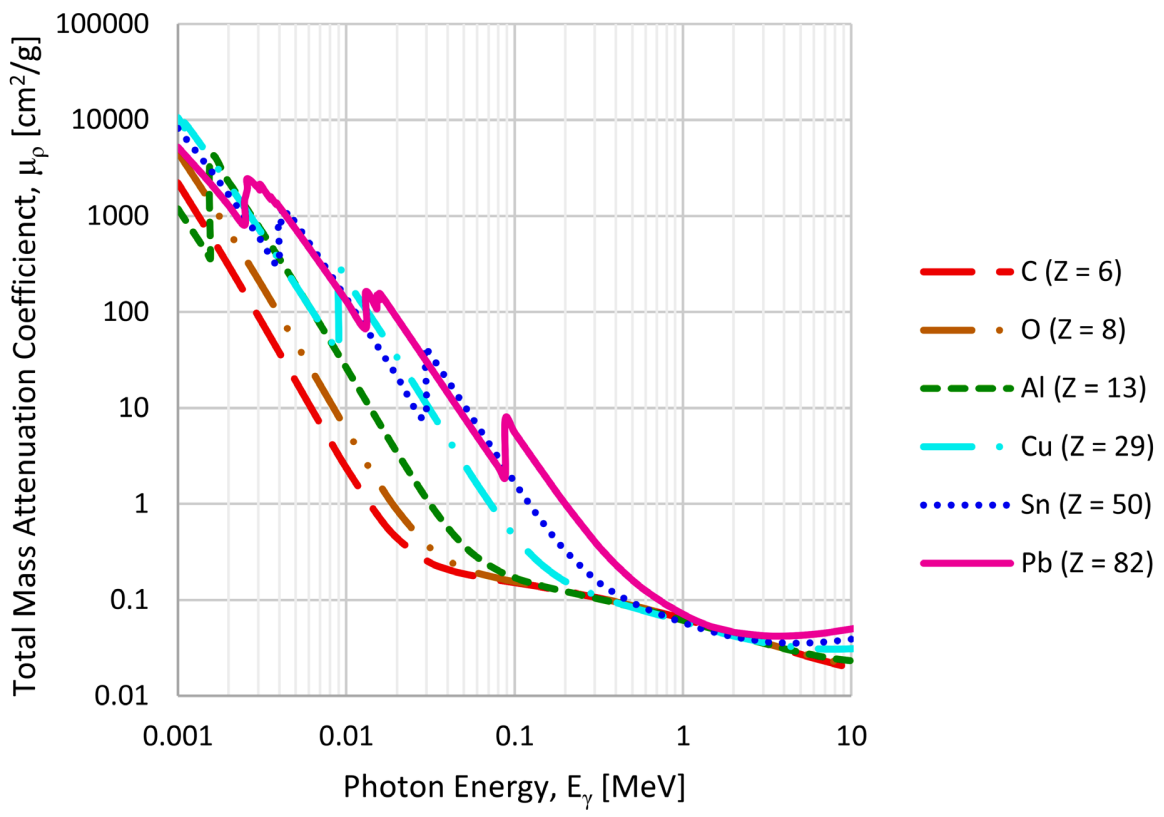

Figure 1. Photon mass attenuation coefficients for select elements of $Z=6$ to 82 extracted from XCOM website [4].

of the medium. On the other hand, as is well known, low-energy photon attenuations are sensitive to the atomic number of the medium as the slope and discontinuities of attenuation coefficients are unique to each element. We, thus, conceive of a system where we may combine the density effect of high-energy photon intensity losses with the atomic number sensitivities of low-energy photons in a single measurement to detect small components of high- $Z$ elements in a given medium. As we perform relative intensity measurements with respect to a reference medium devoid of the contaminants under investigation, our results are free from the variation of detection efficiencies, source strengths and/or source-absorber-detector geometries.

\section{Experimental Method}

\subsection{Choice of Radio Isotopes}

To be able to exploit the dual sensitivities, i.e. a region of high energies $\left(E_{\gamma}>1 \mathrm{MeV}\right)$ where the attenuations depend on the bulk of the medium and the low energies where the elemental constituents reveal themselves, an arrangement of radioactive source(s) emitting photons over a wide range of discrete energies is suggested. In this arrangement, a measurement of relative intensities of the full-energy peaks of the sample in question with a reference sample is made. A detector system of moderate energy resolution will suffice.

We used ${ }^{152} \mathrm{Eu}\left(T_{1 / 2}=13.5\right.$ years $)$ and ${ }^{133} \mathrm{Ba}\left(T_{1 / 2}=10.55\right.$ years $)$ as the pair of gamma-ray sources. Table 1 lists the energies and intensities $(>2 \%)$ of the gamma rays emitted by these isotopes ${ }^{1} .{ }^{152} \mathrm{Eu}$ emits five gamma rays $(779,964,1086$,

${ }^{1}$ The nuclear data is available at the Brookhaven National Laboratory website. https://www.nndc.bnl.gov/. Accessed 29 July 2021. 
Table 1. Energies and intensities $\left(I_{\gamma}>2 \%\right)$ of gamma rays emitted by the ${ }^{152} \mathrm{Eu}$ and ${ }^{133} \mathrm{Ba}$ isotopes [6].

\begin{tabular}{lcc}
\hline & \multicolumn{2}{c}{ Gamma Ray Emissions } \\
\cline { 2 - 3 } Isotope & Energy, $E_{\gamma}[\mathrm{MeV}]$ & Intensity, $I_{\gamma}[\%]$ \\
\hline Ba-133 & 0.0810 & 35.6 \\
Eu-152 & 0.1218 & 28.5 \\
Eu-152 & 0.2447 & 7.5 \\
Ba-133 & 0.2764 & 7.2 \\
Ba-133 & 0.3029 & 18.3 \\
Eu-152 & 0.3443 & 26.5 \\
Ba-133 & 0.3567 & 62.0 \\
Ba-133 & 0.3839 & 8.9 \\
Eu-152 & 0.4114 & 2.2 \\
Eu-152 & 0.4440 & 3.0 \\
Eu-152 & 0.7789 & 12.9 \\
Eu-152 & 1.408 & 4.2 \\
Eu-152 & 0.8674 & 14.5 \\
Eu-152 & 0.9641 & 10.1 \\
Eu-152 & 1.086 & 20.9 \\
Eu-152 & 1.112 & \\
\hline & & \\
\hline
\end{tabular}

$1112,1408 \mathrm{keV}$ energies) of high intensities that can be used to judge the interaction thickness $\rho x\left[\mathrm{~g} / \mathrm{cm}^{2}\right]$ of the material. ${ }^{133} \mathrm{Ba}$ and ${ }^{152} \mathrm{Eu}$ together emit 10 high-intensity gamma rays in the range of 81 to $444 \mathrm{keV}$ which can be used to determine the $Z$ of the material. The $81 \mathrm{keV}$ gamma ray proved to be very useful as it, along with the $121 \mathrm{keV}$ gamma ray of ${ }^{152} \mathrm{Eu}$, overlap with the absorption edges of high- $Z$ elements and their attenuations are sensitive to the presence of mercury, lead and transuranic elements, as we discuss below.

\subsection{Experimental Setup}

Figure 2 shows the top view of the experimental setup; consisting of a high-resolution HPGe (high-purity germanium) gamma-ray detector, a $50 \mathrm{~cm}$ long trough to hold absorber materials, and the ${ }^{133} \mathrm{Ba},{ }^{152} \mathrm{Eu}$ radioactive sources. Each source is point-like and of about $5 \mu \mathrm{Ci}(185 \mathrm{kBq})$ activity. The geometry is such that the detector sees only those gamma rays passing through the absorber medium. During data taking, the detector was shielded with $10 \mathrm{~cm}$ lead blocks to minimize the room background contributions.

To mimic a suitcase of interest for security checks at airports, etc., small bricks of organic material were made by compressing cotton cloths with a hydraulic press to approximately one third of their original size (blocks in Figure 2). Each 


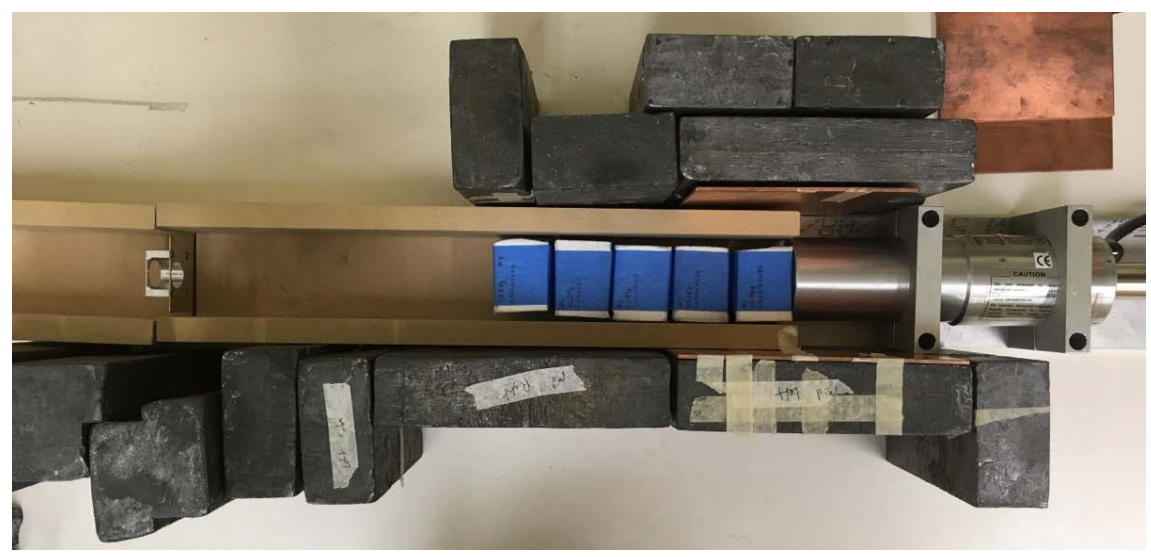

Figure 2. Top view of the Experimental Setup. Left to right: source holder, trough with organic blocks, HPGe detector.

block of organic material had an average density of $0.4 \mathrm{~g} / \mathrm{cm}^{3}$. The overall density thickness of blocks is chosen to correspond to an average suitcase of about 30 $\mathrm{kg}$ in weight and dimensions usually stipulated by the commercial airlines. Rough measurements indicate that moderately packed cotton material has a density of about $0.3 \mathrm{~g} / \mathrm{cm}^{3}$. A common dimension for a large passenger suitcase is $70 \mathrm{~cm}$ tall, $46 \mathrm{~cm}$ wide, and $30 \mathrm{~cm}$ in depth. A density of $0.3 \mathrm{~g} / \mathrm{cm}^{3}$ and dimensions of $(70 \times 46 \times 30) \mathrm{cm}$ corresponds to $29 \mathrm{~kg}$. Most standard check-in baggage allowances are a maximum of $32 \mathrm{~kg}$. Assuming moderate packing, the density thickness through the depth of the suitcase is $9 \mathrm{~g} / \mathrm{cm}^{2}$ and the density thickness through the width of the suitcase is $13.8 \mathrm{~g} / \mathrm{cm}^{2}$. The organic cloths used were made up of unknown proportions of cotton (cellulose $\mathrm{C}_{6} \mathrm{H}_{10} \mathrm{O}_{5}$ ) and polypropylene $\left(\mathrm{C}_{3} \mathrm{H}_{6}\right)$. During the experiment foreign elements of our interest were inserted in between the blocks. The data acquisition system comprised of conventional NIM electronics and an ORTEC-Maestro ADC based program².

\section{Data Analyses}

When calculating theoretical values of attenuation coefficients for the simulated suitcase materials from XCOM database, a 50\% cotton (cellulose), 50\% polypropylene mixture was assumed. To ensure that such assumption is not erroneous, we made XCOM [4] calculations for pure cellulose, pure polypropylene and 50\% admixtures of each of them. As seen in Figure 3, for 81 to $1408 \mathrm{keV}$ photon energies of our interest, the difference in mass attenuation coefficients between the three materials is monotonous and less than $\sim 3 \%$. Clearly, these small variations of attenuation coefficients for possible uncertainties in the relative compositions of cotton and polypropylene are of no consequence for the data analyses and interpretations.

The organic and absorber materials were measured to determine their linear $x[\mathrm{~cm}]$ and density-thicknesses $\rho x\left[\mathrm{~g} / \mathrm{cm}^{2}\right]$. The reference spectrum was ${ }^{2}$ See ORTEC-Maestro User's Manual for information: https://web.archive.org/web/20211002065539/https://www.ortec-online.com/-/media/ametekortec/ manuals/a65-mnl.pdf 


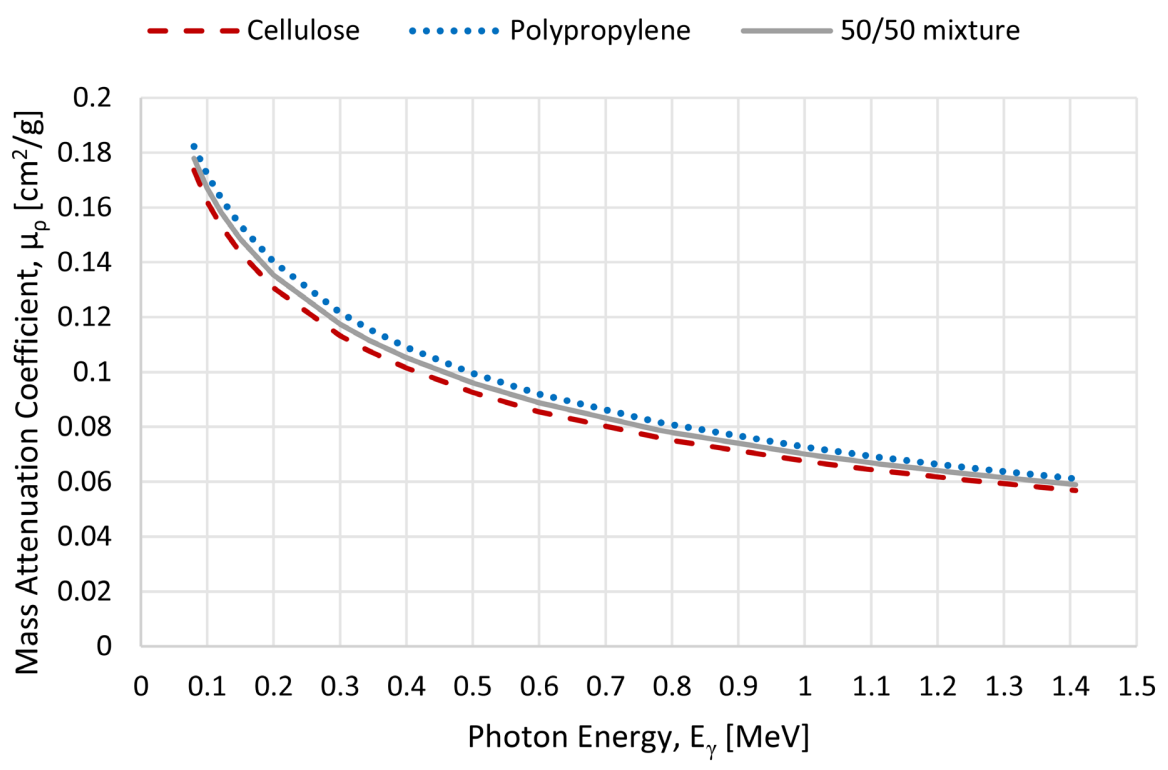

Figure 3. Mass Attenuation Coefficients for pure cellulose, pure polypropylene, and 50-50 admixtures in the organic material (calculated from the XCOM interactive database [4]).

obtained with only organic blocks between the radioactive sources and the detector. The absorber spectra were measured by placing various low- $Z$ (graphite, aluminum), medium- $Z$ (copper, tin), and high- $Z$ (lead) materials in the trough along with the organic blocks. The spectra were collected such that the error in the counts of each peak measurement was less than $2 \%$.

\subsection{Determination of Absorber Role on Intensities}

The number of gamma rays of a certain energy, $E_{\gamma}$, seen by the detector depends on three factors:

1) Yield, $Y\left(E_{\gamma}\right)$-characteristic of decay modes of the isotope, which determine the branching ratios for the intensities of photons of energy $E_{\gamma}$. It also depends on the activity of the source (expressed in Becquerels [Bq] or Curies [Ci]) and the source-medium-detector geometry. In our experiment, the $50 \mathrm{~cm}$ separation between the detector and source was maintained constant throughout.

2) Attenuation-characteristic of the absorbers placed between the detector and the source. This parameter depends on the material constituents of the medium, both for their elemental composition and their thickness $x_{i}$, density $\rho_{i}$, and attenuation coefficient $\mu_{\rho}\left(E_{\gamma}, Z_{i}\right)$, which as seen in Figure 1 is specific to photon energy $E_{\gamma}$. The mass attenuation coefficient of any compound or mixture can be calculated from XCOM data for elements and by assuming Bragg additivity applies, written as [5]:

$$
\mu_{\rho}\left(E_{\gamma}\right)=\sum_{i} w_{i} \cdot \mu_{\rho}\left(E_{\gamma}, Z_{i}\right)
$$

where $w_{i}$ is the fraction by weight of the $I^{\text {th }}$ atomic constituent and $\mu_{\rho}\left(E_{\gamma}, Z_{i}\right)$ is the corresponding mass attenuation of the $I^{\text {th }}$ element. The mass attenuation 
coefficient of a compound or mixture is the sum weighted by the composition. For a gamma ray of energy $E_{\gamma}$, passing through multiple absorbers, each of attenuation coefficient $\mu_{\rho}\left(E_{\gamma}, Z_{i}\right)$, the overall attenuation is given by:

$$
\mathrm{e}^{-\sum_{i} \mu_{\rho}\left(E_{\gamma}, Z_{i}\right) \cdot x_{i} \rho_{i}}
$$

where $x_{i} \rho_{i}$ is the density thickness of th $I^{\text {th }}$ absorber in the setup.

3) Full energy peak efficiency, $\varepsilon\left(E_{\gamma}\right)$-depends on the intrinsic detection efficiency of the detector, which varies with photon energy. The net area of an observed full-energy peak $Y\left(E_{\gamma}\right)$ is then:

$$
Y\left(E_{\gamma}\right)=I\left(E_{\gamma}\right) \varepsilon\left(E_{\gamma}\right) \mathrm{e}^{-\sum_{i} \mu_{\rho}\left(E_{\gamma}, Z_{i}\right) \cdot x_{i} \rho_{i}}
$$

The ratio of areas of two full-energy peaks at energy $E_{\gamma}$ collected using absorbers 1 and 2 maybe written as:

$$
\frac{\left[Y\left(E_{\gamma}\right)\right]_{1}}{\left[Y\left(E_{\gamma}\right)\right]_{2}}=\frac{I\left(E_{\gamma}\right) \varepsilon\left(E_{\gamma}\right)\left[\mathrm{e}^{-\sum_{i} \mu_{\rho}\left(E_{\gamma}, Z_{i}\right) \cdot x_{i} \rho_{i}}\right]_{1}}{I\left(E_{\gamma}\right) \varepsilon\left(E_{\gamma}\right)\left[\mathrm{e}^{-\sum_{j} \mu_{\rho}\left(E_{\gamma}, Z_{j}\right) \cdot x_{j} \rho_{j}}\right]_{2}}
$$

which reduces to:

$$
\frac{\left[Y\left(E_{\gamma}\right)\right]_{1}}{\left[Y\left(E_{\gamma}\right)\right]_{2}}=\frac{\left[\mathrm{e}^{-\sum_{i} \mu_{\rho}\left(E_{\gamma}, Z_{i}\right) \cdot x_{i} \rho_{i}}\right]_{1}}{\left[\mathrm{e}^{-\sum_{j} \mu_{\rho}\left(E_{\gamma}, Z_{j}\right) \cdot x_{j} \rho_{j}}\right]}
$$

where the subscripts 1 and 2 refer to the two spectra with absorber 1 and 2, respectively. This ratio does not depend on the intensity $I\left(E_{\gamma}\right)$ of the gamma ray nor on the full-energy peak detection efficiency $\varepsilon\left(E_{\gamma}\right)$. Thus, it is simply the statistical errors of detected events which enter into the final result. The peak yield in the numerator of the ratio (spectrum 1) is referred to as the absorber spectrum: it is of the spectrum of gamma rays passing through the medium under investigation. The peak yield in the denominator (spectrum 2) is referred to as the reference spectrum, which is devoid of high- $Z$ materials.

An experimental reference spectrum (spectrum 2 in Equations (5) and (6)) was measured by placing materials in the trough that are "expected" to be there. In the case of airport security, organic material (clothes, plastic, etc.) ought to be expected. The experimental ratio of the areas of peaks between the absorber and reference spectra were then calculated.

Figure 4 shows a spectrum with the reference material (upper) and an absorber spectrum which includes a $0.435 \mathrm{~mm}$ lead sheet (lower). A conspicuous feature is that the $121 \mathrm{keV}$ gamma ray in the spectrum with lead absorber is more attenuated than the $81 \mathrm{keV}$ gamma ray, as compared to their relative intensities in the reference spectrum. This is understood as due to smaller attenuation 


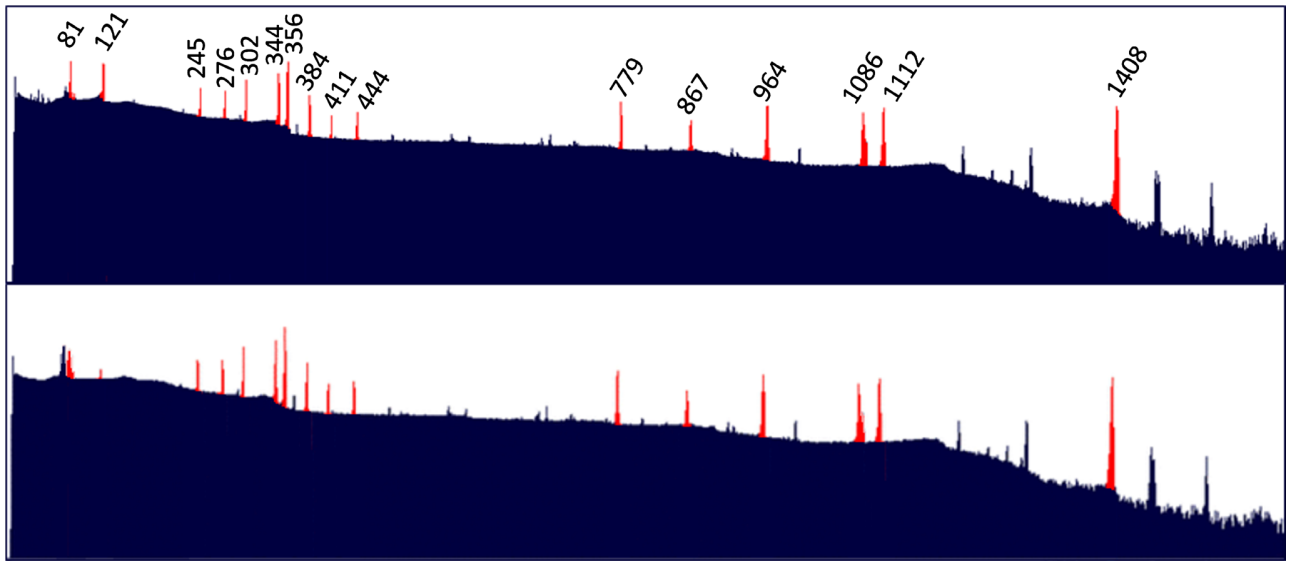

Figure 4. The ${ }^{152} \mathrm{Eu}+{ }^{133} \mathrm{Ba}$ gamma-ray spectrum with reference organic material (upper) and a thin sheet of lead inserted (lower). The 81, 276, 302, 356, $384 \mathrm{keV}$ full-energy peaks are of ${ }^{133} \mathrm{Ba}$ and the other energies are of ${ }^{152} \mathrm{Eu}$ decays. The unmarked peaks near $81 \mathrm{keV}$ are due to $\mathrm{Pb}$ fluorescence, the rest of the unmarked peaks are due to background radiation.

coefficient for $81 \mathrm{keV}$ gamma ray at the lead absorption edge (see Figure 1), while $121 \mathrm{keV}$ gamma ray is outside the absorption edge. All other peaks at higher energies show a monotonous trend for the peak ratios.

The attenuation coefficients of the materials were obtained from XCOM and in some cases, calculated using the Bragg additivity formula (Equation 2). The theoretical ratio of the peaks between the absorber and the reference spectra were then calculated using Equation 6.

\subsubsection{Z-Dependence of the Attenuation}

To examine the Z-dependence of intensity variations, we measured two spectra one each with a copper sheet $\left(0.508 \mathrm{~mm}=0.455 \mathrm{~g} / \mathrm{cm}^{2}\right)$ and one with a lead sheet $\left(0.435 \mathrm{~mm}=0.491 \mathrm{~g} / \mathrm{cm}^{2}\right)$ inserted in the absorber sample. Note that the copper and lead sheets used are of nearly same density thickness. Figure 5 shows the ratio of intensities with respect to the reference absorber (Equation (6)). Also shown are the XCOM results as curves. Two things are worth noting. First, $\mathrm{XCOM}$ calculations are in excellent agreement with the data. Secondly, as expected, for energies above about $800 \mathrm{keV}$, there is no difference between the copper and lead absorbers. At lower energies, the intensities of lead absorber decrease much more steeply than with the copper absorber. Furthermore, the lead absorber shows a minimum ratio at $121 \mathrm{keV}$ with an enhancement at $81 \mathrm{keV}$, not seen in the spectrum with copper sheet. This is understood as due to X-ray absorption edge of lead at $82 \mathrm{keV}$, which is not to be found in copper or other low Z-elements.

\subsubsection{Density Thickness Dependence}

It is of interest to examine the influence of density thickness of organic medium and the level of concentration of foreign materials. To this end, the ratios presented in Figure 6 were collected using absorber samples consisting of $22.1 \mathrm{~cm}$ $\left(8.8 \mathrm{~g} / \mathrm{cm}^{2}\right)$ and $31.0 \mathrm{~cm}\left(12.4 \mathrm{~g} / \mathrm{cm}^{2}\right)$ of organic blocks each with a $0.435 \mathrm{~mm}$ thick lead sheet $\left(0.493 \mathrm{~g} / \mathrm{cm}^{2}\right)$ inserted, and a sample containing $44.9 \mathrm{~cm}(18.0$ 


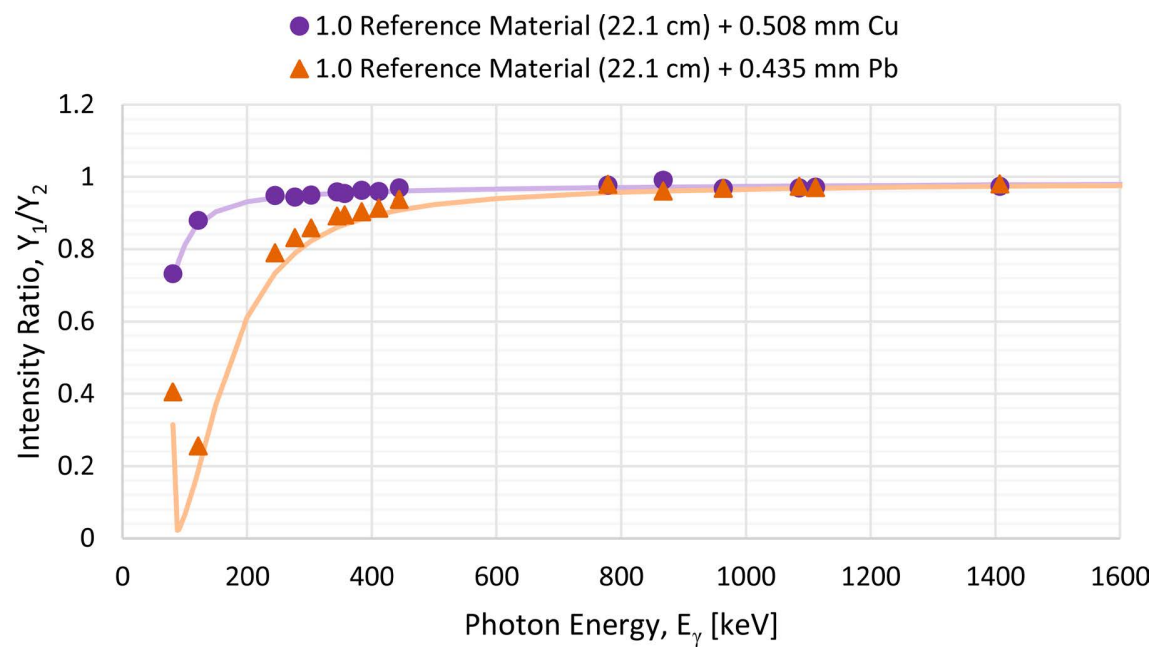

Figure 5. Measured intensity ratios of $22.1 \mathrm{~cm}$ reference material $\left(8.8 \mathrm{~g} / \mathrm{cm}^{2}\right)+0.508 \mathrm{~mm}$ copper $\left(0.455 \mathrm{~g} / \mathrm{cm}^{2}\right)$, and reference + lead sheet $\left(0.493 \mathrm{~g} / \mathrm{cm}^{2}\right)$ with respect to the reference material. Also plotted are the XCOM calculation results. The sensitivity of the ratios to the $\mathrm{Z}$-value of absorber at low energies is clear.

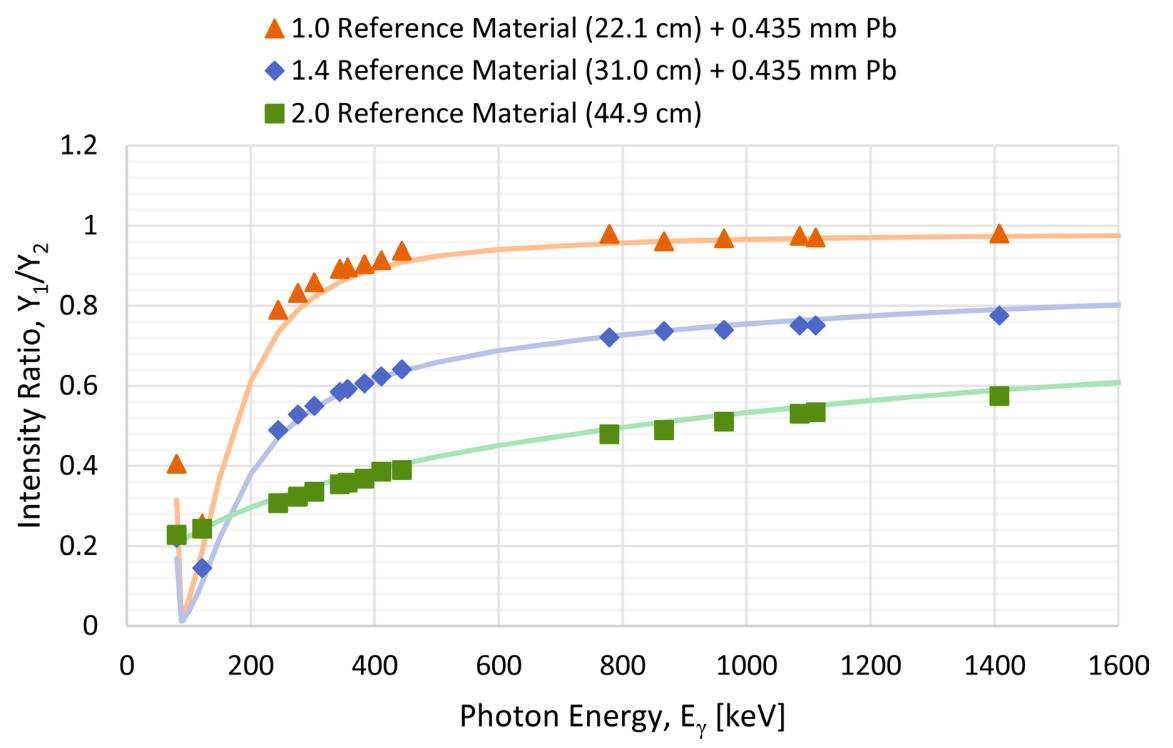

Figure 6. Experimental and theoretical (XCOM) intensity ratios of organic block medium (reference material, $22.1 \mathrm{~cm}, 8.8 \mathrm{~g} / \mathrm{cm}^{2}$ ) and the effect of a thin lead sheet $(0.435 \mathrm{~mm}$, $\left.0.493 \mathrm{~g} / \mathrm{cm}^{2}\right)$ inserted. At each energy, the data are normalized to yields with the reference material: triangles $22.1 \mathrm{~cm}$ organic medium $\left(8.8 \mathrm{~g} / \mathrm{cm}^{2}\right)+$ lead, diamonds $31.0 \mathrm{~cm}$ organic medium $\left(12.4 \mathrm{~g} / \mathrm{cm}^{2}\right)+$ lead, and squares $44.9 \mathrm{~cm}$ organic medium $\left(18.0 \mathrm{~g} / \mathrm{cm}^{2}\right)$. The solid points are experimental data. The curves are corresponding XCOM calculations.

$\mathrm{g} / \mathrm{cm}^{2}$ ) of organic blocks only. The spectrum of $22.1 \mathrm{~cm}$ organic blocks with no lead sheet is used as the reference spectrum. Superposed on the data in Figure 6 are the XCOM calculation results normalized to the $22.1 \mathrm{~cm}$ organic block reference spectrum at each gamma-ray energy.

A few observations of the results are in order:

1) The XCOM and experiment are in excellent agreement. The attenuations for all thicknesses with and without lead sheet are well accounted for. 
2) For photon energies above about $800 \mathrm{keV}$, the ratio with respect to the reference spectrum decreases to $96 \%-98 \%$ for $22.1 \mathrm{~cm}$ organic block + lead, and to $72 \%-78 \%$ level for $31 \mathrm{~cm}$ organic block + lead and to $60 \%$ level with no lead.

3) At photon energies below $400 \mathrm{keV}$, the samples with lead show steeper decrease in the intensity with decreasing energy, while the $44 \mathrm{~cm}$ block without lead sheet has nearly continuous change with no steep decrease.

4) As indicated above, the organic blocks with lead sheet shows enhanced intensity of $81 \mathrm{keV}$ peak relative to $121 \mathrm{keV}$, which is understood as due to an $\mathrm{X}$-ray absorption edge.

It is thus clear that the presence of high- $Z$ materials significantly attenuates the low-energy photons without much change at high energies, while increase of the mass density alone brings down the overall intensities. It is also seen that the high-energy gamma rays show the sensitivity to density thickness $x \rho$ of the absorber. XCOM database shows attenuation coefficients for atomic numbers $Z$ $=1-100$ behave very similarly around $1 \mathrm{MeV}$ but with dramatic variations at low energies.

If the density thickness of two absorbers is same, then their ratio of peak intensities above about $1 \mathrm{MeV}$ will be nearly the same. This is demonstrated in Figure 7, where we plot the XCOM results for $1 \mathrm{~mm}$ thick absorbers of graphite $\left(Z=6, \rho=2.27 \mathrm{~g} / \mathrm{cm}^{3}\right)$, aluminum $\left(Z=13, \rho=2.7 \mathrm{~g} / \mathrm{cm}^{3}\right)$, copper $(Z=29, \rho=$ $\left.8.96 \mathrm{~g} / \mathrm{cm}^{3}\right)$, tin $\left(Z=50, \rho=7.31 \mathrm{~g} / \mathrm{cm}^{3}\right)$, gadolinium $\left(Z=64, \rho=7.90 \mathrm{~g} / \mathrm{cm}^{3}\right)$ and lead $\left(Z=82, \rho=11.34 \mathrm{~g} / \mathrm{cm}^{3}\right)$. The data points in Figure 7 indicate the photon energies of the ${ }^{133} \mathrm{Ba}$ and ${ }^{152} \mathrm{Eu}$ sources. As seen in Figure 7, the intensities of spectra with copper $\left(Z=29, \rho=8.96 \mathrm{~g} / \mathrm{cm}^{3}\right)$, tin $\left(Z=50, \rho=7.31 \mathrm{~g} / \mathrm{cm}^{3}\right)$, and gadolinium $\left(Z=64, \rho=7.90 \mathrm{~g} / \mathrm{cm}^{3}\right)$ absorbers are within $1 \%$ at about 1 $\mathrm{MeV}$, while the three spectra clearly separate the three distinct materials at low energies below about $200 \mathrm{keV}$. This confirms that the high-energy gamma rays

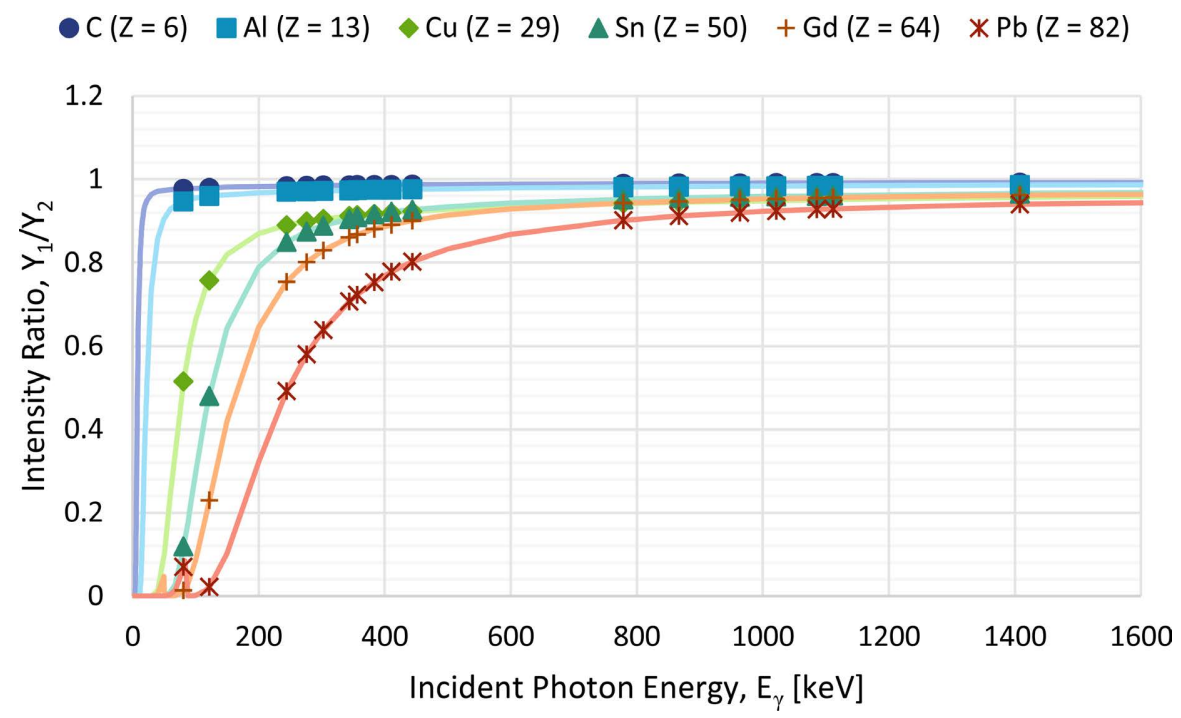

Figure 7. Theoretical XCOM calculations of ratios of peak intensities per $1 \mathrm{~mm}$ of absorber elements. Data points are ${ }^{133} \mathrm{Ba}$ and ${ }^{152} \mathrm{Eu}$ gamma-ray energies. 
are insensitive to changes in $Z$. If the data is taken using solely high-energy or low-energy gamma rays, the effects due to different densities and different $Z$ numbers are indiscernible. The wide energy range of gamma rays emitted by the ${ }^{133} \mathrm{Ba}$ and ${ }^{152} \mathrm{Eu}$ sources make the evaluation of both the $Z$-number and density of the absorbing material possible.

\section{Calculations for Transuranic Elements and Mercury}

While we did not perform experiments with uranium, plutonium, or mercury for logistic reasons, we extend our calculations of XCOM for plutonium and mercury, representing a security and a health risk, respectively.

\subsection{Presence of Plutonium as Trace Element}

Plutonium $(Z=94)$ and uranium $(Z=92)$ are neighboring elements and solid materials. Our calculation for plutonium is readily applicable to uranium and nearby transuranic elements such as americium. We use the same reference material (organic block of $22.1 \mathrm{~cm}$ ) to examine the response to plutonium as foreign element. In Figure 8 we see an indication of a high- $Z$ material as an X-ray absorption edge kink, even with plutonium of 10-micron thickness. The 100-micron foil will show the characteristic X-ray absorption structure. For larger thicknesses, the steep drop of intensities below $500 \mathrm{keV}$ is a clear indication of the presence of high $Z$-materials.

\subsection{Presence of Mercury as a Contaminant}

Globally, there is a serious concern of mercury contamination in water as a hazardous substance [6] likely to cause serious health effects. About 128 countries signed legally binding agreements to limit mercury concentrations. We explored

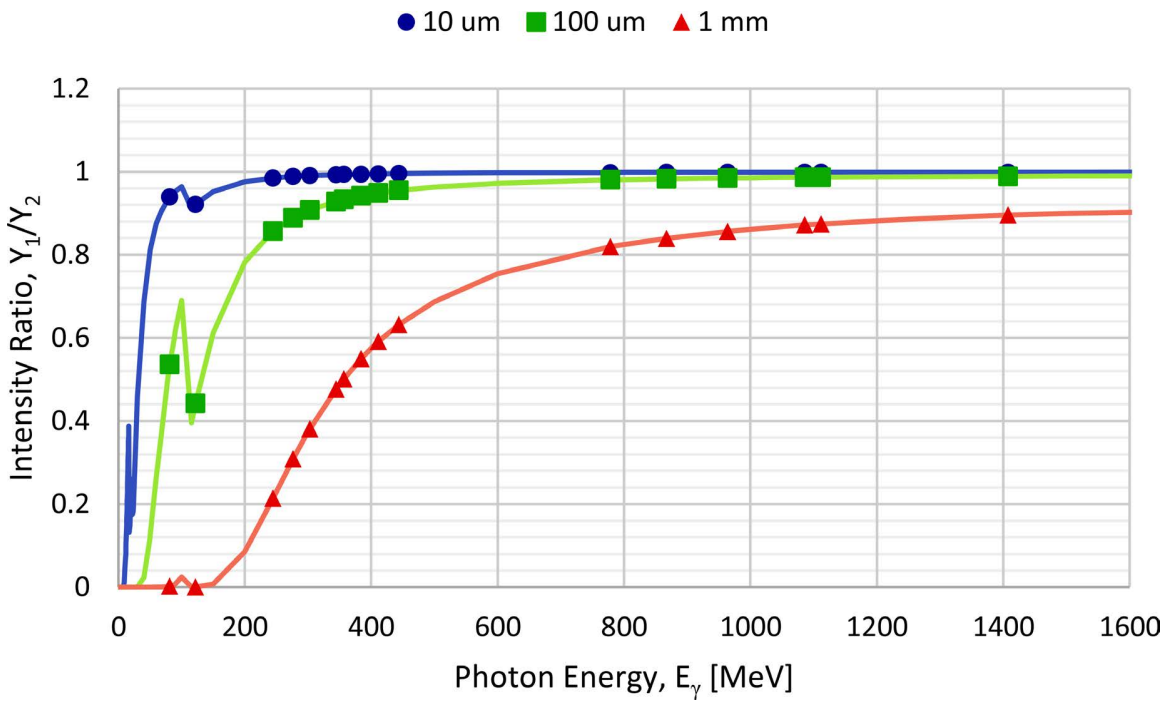

Figure 8. XCOM calculations for attenuation of ${ }^{133} \mathrm{Ba}$ and ${ }^{152} \mathrm{Eu}$ gamma rays for various plutonium thicknesses embedded in the reference material. The $\mathrm{Pu}$ content: circles $(10$ microns, $\left.0.02 \mathrm{~g} / \mathrm{cm}^{2}\right)$, squares $\left(100\right.$ microns, $\left.0.2 \mathrm{~g} / \mathrm{cm}^{2}\right)$, and triangles $\left(1 \mathrm{~mm}, 2 \mathrm{~g} / \mathrm{cm}^{2}\right)$. 
the possibility that multi-energy gamma-ray attenuation techniques could be a potential tool for detecting low concentrations of mercury. The occurrence of mercury in aqueous solutions, say water, is a volume effect and one must treat it as a mixture. One concerns here with concentrations less than one part per billion (ppb) in water. The X-ray absorption edge of mercury is close to ${ }^{133} \mathrm{Ba}$ 's 81 $\mathrm{keV}$ gamma ray. Figure 9 shows the results of XCOM calculations for $1 \mathrm{cc}$ water with admixtures of $0.001 \%-1 \%$ of mercury by volume. While the arrangement can detect concentrations of higher than $0.1 \%$ (one part in 1000), or better, it became obvious that this technique is not competitive against other technologies which can detect contamination levels of much less than a few parts in billion [6] [7].

\section{Discussion}

The experiment and XCOM calculations clearly indicate that a simultaneous measurement of gamma-ray intensity attenuations over a range of energies, about 80 to $1500 \mathrm{keV}$, can discern the presence of high- $Z$ elements in otherwise dense medium. The test arrangement can be easily achieved with the simultaneous use of two radioactive isotopes of a few years lifetimes.

Below we compare the sensitivity of this method to that of more commonly used techniques such as the continuous spectrum of low-energy X-rays and nearly monoenergetic high-energy gamma rays. X-ray tubes provide a continuous energy spectrum of photons below about $200 \mathrm{keV}$ and have long been used to identify materials. While the low-energy photons are very sensitive to the atomic number of a medium, they are easily attenuated. Table 2 compares the response of the low-energy photons to that of ${ }^{152} \mathrm{Eu}$ emissions for media of $20 \mathrm{~cm}$ thickness. The low transmission of X-ray energies is its limiting factor for use in

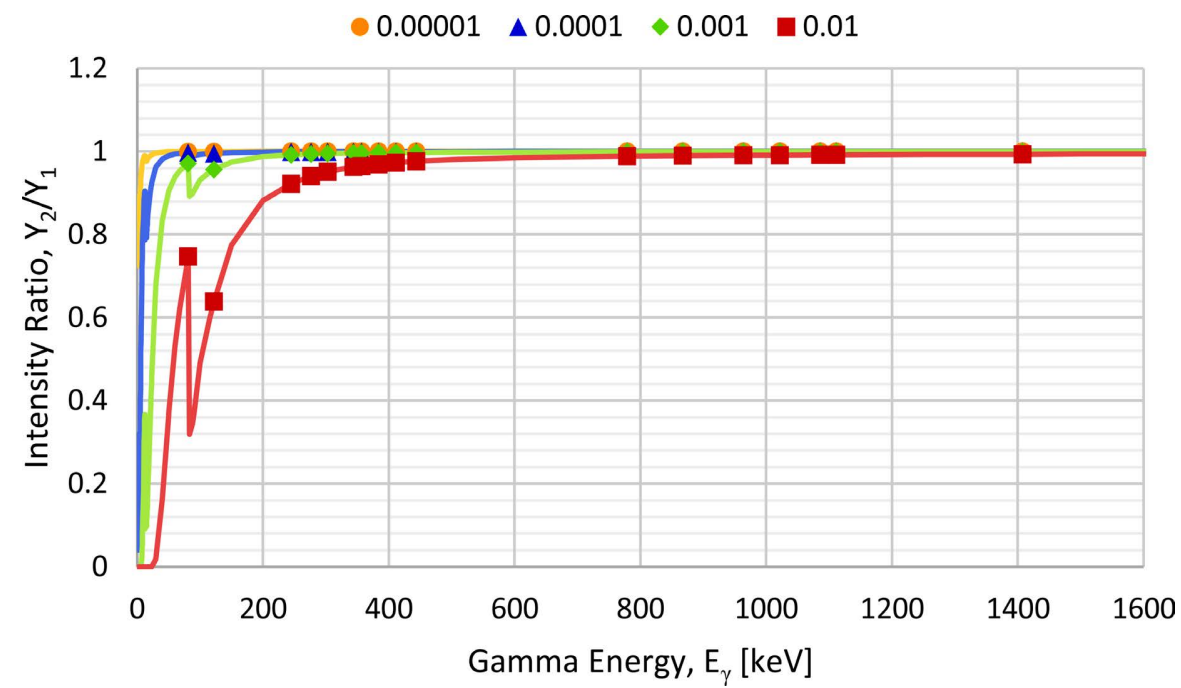

Figure 9. XCOM calculations for attenuation of ${ }^{133} \mathrm{Ba}$ and ${ }^{152} \mathrm{Eu}$ gamma rays through a 1 cc water column, mercury contaminants indicated by volume $[\mathrm{mL} / \mathrm{mL}]$. Hg concentrations: circles $10^{-5}[\mathrm{~mL} / \mathrm{mL}]$, triangles $10^{-4}[\mathrm{~mL} / \mathrm{mL}]$, diamonds $10^{-3}[\mathrm{~mL} / \mathrm{mL}]$, square $10^{-2}$ $[\mathrm{mL} / \mathrm{mL}]$. 
bulky media.

In comparison to ${ }^{152} \mathrm{Eu}$ gamma rays, the $\mathrm{X}$-ray tube would have to be run for almost a factor of three times longer for low- $Z$ materials (C-6, Al-13) and many orders of magnitude longer for medium to high- $Z$ materials in order to achieve the same sensitivity. For X-ray tubes producing photons at energies lower than $200 \mathrm{keV}$, the run times will be even longer. The US military uses a cobalt-60 $\left({ }^{60} \mathrm{Co}\right)$ source to probe vehicles ${ }^{3}$. This isotope emits two gamma rays of nearly the same energy (1173 and $1333 \mathrm{keV}$ ) which, as we have seen above, are insensitive to the atomic number $Z$ of absorber elements. In Table 3, the transmission of gamma rays produced by ${ }^{133} \mathrm{Ba},{ }^{152} \mathrm{Eu}$, and ${ }^{60} \mathrm{Co}$ sources through $1 \mathrm{~mm}$ of various materials is calculated. It is seen that the relative transmission for $\mathrm{C}: \mathrm{Al}: \mathrm{Cu}: \mathrm{Sn}: \mathrm{Pb}$ are 1.0:0.998:0.962:0.977:0.95 and 1.0:0.98:0.523:0.113:0.066 respectively for 1333 and $81 \mathrm{keV}$ gamma rays. This observation clearly illustrates that the high-energy $\left(E_{\gamma}>1 \mathrm{MeV}\right)$ gamma rays are not sensitive to the medium's atomic number.

Table 2. Transmission of $200 \mathrm{keV} \mathrm{X}$-rays and select ${ }^{152} \mathrm{Eu}$ gamma rays through a $20 \mathrm{~cm}$ thick sample of select materials.

\begin{tabular}{ccccccc}
\hline \multirow{5}{*}{ Source } & \multicolumn{5}{c}{ Transmission Through $20 \mathrm{~cm}$ of Material } & $\mathrm{e}^{-(\mu(E, Z) \cdot \cdot 20[\mathrm{~cm}])}$ \\
\cline { 2 - 7 } & $\begin{array}{c}\text { Energy, } E \\
{[\mathrm{keV}]}\end{array}$ & $\begin{array}{c}\mathrm{C} \\
(Z=6)\end{array}$ & $\begin{array}{c}\mathrm{Al} \\
(Z=13)\end{array}$ & $\begin{array}{c}\mathrm{Cu} \\
(Z=29)\end{array}$ & $\begin{array}{c}\mathrm{Sn} \\
(Z=50)\end{array}$ & $\begin{array}{c}\mathrm{Pb} \\
(Z=82)\end{array}$ \\
\hline X-ray Tube & 200 & $3.80 \mathrm{e}-3$ & $1.35 \mathrm{e}-3$ & $7.36 \mathrm{e}-13$ & $2.13 \mathrm{e}-21$ & $4.36 \mathrm{e}-99$ \\
${ }^{152} \mathrm{Eu}$ & 344 & $1.01 \mathrm{e}-2$ & $4.84 \mathrm{e}-3$ & $1.02 \mathrm{e}-8$ & $2.06 \mathrm{e}-9$ & $6.04 \mathrm{e}-31$ \\
${ }^{152} \mathrm{Eu}$ & 779 & $1.48 \mathrm{e}-2$ & $1.48 \mathrm{e}-2$ & $1.16 \mathrm{e}-6$ & $7.17 \mathrm{e}-6$ & $5.10 \mathrm{e}-13$ \\
${ }^{152} \mathrm{Eu}$ & 1086 & $6.25 \mathrm{e}-2$ & $4.12 \mathrm{e}-2$ & $3.96 \mathrm{e}-5$ & $3.16 \mathrm{e}-4$ & $3.16 \mathrm{e}-7$ \\
${ }^{152} \mathrm{Eu}$ & 1408 & $6.47 \mathrm{e}-2$ & $4.28 \mathrm{e}-2$ & $4.50 \mathrm{e}-5$ & $3.55 \mathrm{e}-4$ & $4.35 \mathrm{e}-7$ \\
\hline
\end{tabular}

Table 3. Transmission of ${ }^{60} \mathrm{Co}$ and select ${ }^{133} \mathrm{Ba},{ }^{152} \mathrm{Eu}$ gamma rays through a $1 \mathrm{~mm}$ thick sample of select materials.

\begin{tabular}{|c|c|c|c|c|c|c|}
\hline \multirow{2}{*}{ Source } & \multicolumn{6}{|c|}{ Transmission Through $1 \mathrm{~mm}$ of Material $\mathrm{e}^{-(\mu(E, Z) \cdot \rho \cdot 0.1[\mathrm{~cm}])}$} \\
\hline & $\begin{array}{c}\text { Energy, } E \\
{[\mathrm{keV}]}\end{array}$ & $\begin{array}{c}\mathrm{C} \\
(Z=6)\end{array}$ & $\begin{array}{c}\mathrm{Al} \\
(Z=13)\end{array}$ & $\begin{array}{c}\mathrm{Cu} \\
(Z=29)\end{array}$ & $\begin{array}{c}\text { Sn } \\
(Z=50)\end{array}$ & $\begin{array}{c}\mathrm{Pb} \\
(Z=82)\end{array}$ \\
\hline${ }^{133} \mathrm{Ba}$ & 81 & 0.9642 & 0.9469 & 0.5048 & 0.1096 & 0.0644 \\
\hline${ }^{152} \mathrm{Eu}$ & 122 & 0.9680 & 0.9596 & 0.7543 & 0.4725 & 0.0203 \\
\hline${ }^{152} \mathrm{Eu}$ & 1086 & 0.9862 & 0.9842 & 0.9506 & 0.9605 & 0.9279 \\
\hline${ }^{152} \mathrm{Eu}$ & 1112 & 0.9864 & 0.9844 & 0.9512 & 0.9611 & 0.9294 \\
\hline${ }^{60} \mathrm{Co}$ & 1173 & 0.9868 & 0.9848 & 0.9525 & 0.9622 & 0.9324 \\
\hline${ }^{60} \mathrm{Co}$ & 1333 & 0.9876 & 0.9857 & 0.9554 & 0.9647 & 0.9383 \\
\hline${ }^{152} \mathrm{Eu}$ & 1408 & 0.9879 & 0.9861 & 0.9566 & 0.9657 & 0.9404 \\
\hline
\end{tabular}

${ }^{3}$ See for example, Science Applications International Corporation (SAIC) website: https://web.archive.org/web/20071011181039/http://www.saic.com/products/security/pdf/mobile-V ACIS.pdf 
While the low-energy photons are sensitive to atomic number, the high attenuation renders them impractical for dense materials. The advantage of a multi-energy photon arrangement for energies of about $80 \mathrm{keV}$ to $1.4 \mathrm{MeV}$ to discriminate the contribution from high- $Z$ materials versus high density materials is clear.

\section{Conclusion}

From experiment and XCOM calculations we demonstrated that a radioactive source arrangement of multi-energy photons can detect small quantities of high- $Z$ contraband materials in otherwise low- $Z$ media, such as suitcases. While the high- $Z$ contaminants such as mercury in water or other environmental samples can also be detected, nano technology [7] and diverse analytical methods [6] may be more competitive than the gamma-ray attenuation methods. In comparison to X-ray tubes or particle accelerators, the radioactive source arrangement has practical advantages in that the sources are fail-proof, continuously operating systems, and require very little maintenance. Once procured, they can operate for years without additional investment due to their long half-lives. On the other hand, X-ray machines or accelerators require substantial maintenance, repairs, and elaborate power supply schemes; high-energy photon machines require large infrastructures. As an example, an X-ray scanner comprises of an $\mathrm{X}$-ray tube and an X-ray detector, both of which consume power and require regular maintenance. An X-ray tube running at $100 \mathrm{kVp}$ and $100 \mathrm{~mA}$ would consume at least $10 \mathrm{~kW}$. It is safe to assume that the entire X-ray tube system would consume much more power. In our set-up of radioactive sources and a detector, only the detector and electronic assembly consume power, which can be drawn from a wall power outlet. The ${ }^{133} \mathrm{Ba}$ and ${ }^{152} \mathrm{Eu}$ sources only need to be replaced once or twice every half-life $\left(T_{1 / 2}=10.55 \mathrm{y}\right.$ for ${ }^{133} \mathrm{Ba}, T_{1 / 2}=13.52 \mathrm{y}$ for $\left.{ }^{152} \mathrm{Eu}\right)$. Also, a combination of ${ }^{154} \mathrm{Eu}\left(T_{1 / 2}=8.59 \mathrm{y}\right)$ emitting six gamma rays of $123-1205 \mathrm{keV}$ energies along with ${ }^{133} \mathrm{Ba}$ can be a useful pair of isotopes. Of course, one can employ more than two sources without loss of information. Needless to say, fewer the number of sources, easier it is to handle. While we performed these tests with a single detector in a laboratory setting, the technique can be easily extended to a multi-detector ring system for tomographic reconstructions. In the field implementation, this system can be operated by a person or at most a few people.

\section{Acknowledgements}

This research was partially funded by a discovery research grant of Natural Sciences and Engineering Council (NSERC) of Canada to Chary Rangacharyulu.

\section{Conflicts of Interest}

The authors declare no conflicts of interest regarding the publication of this paper. 


\section{References}

[1] Rai, P.K., Lee, S.S., Zhang, M., Tsang, Y.F. and Kim, K. (2019) Heavy Metals in Food Crops: Health Risk, Fate, Mechanisms, and Management. Environmental International, 125, 365-385. https://doi.org/10.1016/j.envint.2019.01.067

[2] Van Liew, S., Bertozzi, W., D’Olympia, N., Franklin, W.A., Korbly, S.E., Ledoux, R.J. and Wilson, C.M. (2017) Identification and Imaging of Special Nuclear Materials and Contraband Using Active X-ray Interrogation. Physics Procedia, 90, 313-322. https://doi.org/10.1016/j.phpro.2017.09.023

[3] Ohgaki, H., Daito, I., Zen, H., Kii, T., Masuda, K., Misawa, T., Hajima, R., Hayakawa, T., Shizuma, T., Kando, M. and Fujimoto, S. (2017) Nondestructive Inspection System for Special Nuclear Material Using Inertial Electrostatic Confinement Fusion Neutrons and Laser Compton Scattering Gamma-Rays. IEEE Transactions on Nuclear Science, 64, 1635-1640. https://doi.org/10.1109/TNS.2017.2652619

[4] Berger, M.J., et al. (2010) XCOM: Photon Cross Sections Database. NIST Standard Reference Database 8 (XGAM). NIST, PML, Radiation Physics Division. https://dx.doi.org/10.18434/T48G6X

[5] Rangacharyulu, C. (2014) Physics of Nuclear Radiations-Concepts, Techniques and Applications, Taylor \& Francis. CRC Press, Boca Raton. https://doi.org/10.1201/b16330

[6] Bravo, A.A., Kothawala, D.N., Attermeyer, K., Tessier, E., Bodmer, P. and Amouroux, D. (2018) Clearing and Sampling Protocol for Analysis of Mercury and Dissolved Organic Matter in Freshwater Systems. MethodX, 5, 1017-1026.

https://doi.org/10.1016/j.mex.2018.08.002

[7] Wang, G., Lim, C., Chen, L., Chou, H., Choo, J., Hong, J. and de Mello, A.J. (2009) Surface Enhanced Raman Scattering in Nanoliter Droplets: Towards High Sensitivity Detection of Mercury (II). Analytical and Bioanalytical Chemistry, 394, 1827-1832. https://doi.org/10.1007/s00216-009-2832-7 